\title{
CRISPR-Click Enables Multi-Gene Editing with Modular Synthetic sgRNAs
}

Hansol Park, ${ }^{\text {a\# }}$ Eiman A. Osman, ${ }^{\text {a\# }}$ Christopher R. Cromwell, ${ }^{\text {b }}$ Christopher D. St. Laurent, ${ }^{\text {a }}$ Yuning Liu, ${ }^{a}$ Elena N. Kitova, ${ }^{a}$ John S. Klassen, ${ }^{a}$ Basil P. Hubbard, ${ }^{b}$ Matthew S. Macauley, ${ }^{\text {a,c* }}$ Julianne M. Gibbs ${ }^{\text {a* }}$

a Department of Chemistry, ${ }^{b}$ Department of Pharmacology, ${ }^{c}$ Department of Medical Microbiology and Immunology, University of Alberta, Edmonton, Alberta T6G 2G2, Canada.

\#Joint-first authors

*Co-corresponding authors

TOC graphic

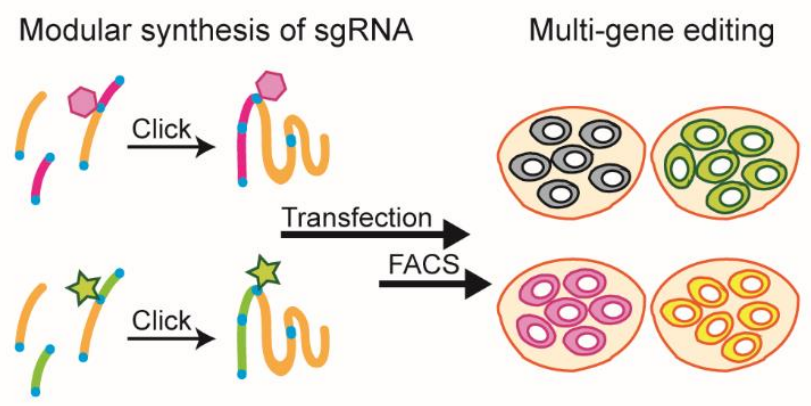




\section{ABSTRACT (250 words; currently 240 words)}

Gene editing systems such as CRISPR/Cas9 readily enable individual gene phenotypes to be studied through loss-of-function. However, in certain instances, gene compensation can obfuscate the results of these studies, necessitating the editing of multiple genes to properly identify biological pathways and protein function. Performing multiple genetic modifications in cells remains difficult due to the requirement for multiple rounds of gene editing. While fluorescently labeled guide RNAs (gRNAs) are routinely used in laboratories for targeting CRISPR/Cas9 to disrupt individual loci, technical limitations in single guide RNA (sgRNA) synthesis hinder the expansion of this approach to multi-color cell sorting. Here, we describe a modular strategy for synthesizing sgRNAs where each target sequence is conjugated to a unique fluorescent label, which enables fluorescence-assisted cell sorting (FACS) to isolate cells that incorporate the desired combination of gene-editing constructs. We demonstrate that three short strands of RNA functionalized with strategically placed 3'-azide and 5'-alkyne terminal deoxyribonucleotides can be assembled in a one-step, template-assisted, copper-catalyzed alkyne-azide cycloaddition (CuAAC) to generate fully functional, fluorophore-modified sgRNAs. Using these synthetic sgRNA in combination with FACS, we achieved selective cleavage of two targeted genes, either separately as a single color experiment or in combination as a dual-color experiment. These data indicate that our strategy for generating doubly-clicked sgRNA allows for Cas9 activity in cells. By minimizing the size of each RNA fragment to 41 nucleotides or less, this strategy is well suited for custom, scalable synthesis of sgRNAs. 


\section{INTRODUCTION}

CRISPR-Cas9 has enabled gene editing both in the lab, ${ }^{1-9}$ allowing for the function of proteins to be readily interrogated, ${ }^{10-12}$ and in the clinic, where it promises to offer treatments for diseases such as sickle cell anemia and cystic fibrosis. ${ }^{13-18}$ Cas9 directs sequence specific DNA cleavage through the aid of a CRISPR RNA (crRNA), which contains a 20-nucleotide (nt) programmable target sequence, and a trans-activating crRNA (tracrRNA) that binds the Cas9. The tracrRNA acts as a bridge between the crRNA and the enzyme. ${ }^{1}$ As CRISPR-Cas9 directed cleavage can vary in efficiency depending on the target, one strategy to ensure gene knockout is to target cutting of the gene at multiple locations by introducing multiple guide RNAs., $3,19,20$ Additionally, other applications in research, as well as clinical applications, require multiple genes to be edited. ${ }^{21-23}$ However, targeting multiple genes through iterative rounds of gene editing requires cells to be cultured for prolonged periods of time, which can be time prohibitive and not feasible for some cell lines. ${ }^{22,23}$ Simultaneous disruption of numerous loci in one round solves these issues.

Several solutions have been advanced with the goal of multiplexing CRISPR-Cas9 to target multiple loci simultaneously. One strategy involves encoding multiple guide RNA (gRNA) via lentiviral transduction ${ }^{24,25}$ or transfection of plasmids containing the appropriate sequences. ${ }^{3,4,20,26,27}$ One particularly elegant example that has been reported is a lentiviral delivery system that uses the Cpf1 nuclease (Cas12) and a self-processing guide RNA to target four different genes. ${ }^{28}$ While these approaches use DNA-encoded strategies, the direct introduction of a gRNA to cells has been shown to have advantages. ${ }^{6,17,29-37}$ For example, direct introduction of synthetic crRNA and tracrRNA made by solid-phase, rather than enzyme-catalyzed synthesis, allows unnatural chemical modifications to be incorporated that confer benefits, such as increased 
stability, ${ }^{31-33}$ reduced off-target effects, ${ }^{34,35}$ and fluorescent labeling. ${ }^{35-37}$ Accordingly, an attractive approach to multiplexing genome editing that alleviates the need for in-cell processing is to introduce multiple gRNAs simultaneously into a cell. This can be achieved by complexing multiple guides using a nanoparticle delivery agent, ${ }^{38,39}$ or introducing multiple gRNAs targeting different genes into the same cell. ${ }^{26,40}$

Synthetic gRNAs can be assembled using two different methods. The first is the dual strand approach, where the crRNA is hybridized to the tracrRNA; for the Cas9 system, these strands are 29-42 and 65-75 nucleotides, respectively. ${ }^{1,41,42}$ Using this dual strand system, chemicallymodified crRNAs such as those incorporating bridged nucleic acids, 2'- $O$-methyl, 2'-fluoro, phosphorothioate, 2'-O-methyl-thiophosphonoacetate, 2'-O-methyl-phosphonoacetate, or 2'-(S)constrained ethyl RNA have been produced with increased activity, ${ }^{31,33,41}$ enhanced stability, ${ }^{31,33,41}$ or decreased off-target cutting. ${ }^{35}$ Likewise, fluorescent modifications to the tracrRNA have been used in combination with FACS to isolate cells taking up RNP (and therefore have a higher probability of gene-target cleavage), which is helpful for cells that are difficult to transfect. ${ }^{43,44}$ In the second synthetic approach, the full crRNA-tracrRNA sequence is combined into one strand resulting in a single-guide RNA (sgRNA) molecule of $~ 97$ nucleotides in length. ${ }^{45}$ It has been reported that sgRNAs synthesized by solid-phase synthesis led to higher efficiencies in gene editing compared to dual crRNA:tracrRNA systems. ${ }^{33,46}$ However, due to challenges in synthesizing chemically-modified RNA strands of this length, it has not commonly been used to introduce chemical modifications in sgRNAs, ${ }^{32-34,46,47}$ such as fluorescent modifications.

One of the main challenges associated with accessing chemically-modified sgRNAs stems from difficulties in synthesizing long RNA by solid-phase synthesis. ${ }^{48-51}$ For example, the solidphase synthesis of a 97 mer sgRNA would be $5.4 \%$ if the theoretical efficiency of each step were 
$97 \%\left(0.97^{96}=0.054\right)$. Recent progress in improving the coupling efficiency of each step in the solid-phase synthesis has allowed sgRNA to be directly produced, ${ }^{32-34}$ but such approaches have not been expanded to introduce fluorescent modifications, which can often exhibit reduced coupling efficiencies. Moreover, the low overall yield - even with high coupling efficiencies on a per-step basis - leads to such small quantities of sgRNA material that they cannot be thoroughly purified or characterized by gel electrophoresis and mass spectrometry. This inability to produce, purify, and characterize sizable amounts of synthetic sgRNA also limits the generation of welldefined hybrid materials that facilitate cellular delivery of multiple gene-editing constructs. ${ }^{52}$

One strategy that has been explored to increase the amount and structural diversity of sgRNA involves synthesizing it from two smaller fragments using enzyme-catalyzed ${ }^{37,53}$ or bioconjugation techniques. ${ }^{50,51}$ The first reported example of the latter involved the introduction of a hexyne phosphoramidite monomer at the 5' end of the tracrRNA and a 3'-amine modification on the crRNA further functionalized with azido-acetic acid NHS ester. ${ }^{50}$ The tracrRNA and crRNA were then 'clicked' together using copper-catalyzed alkyne-azide cycloaddition (CuAAC) chemistry. ${ }^{50}$ The resulting sgRNA was capable of directing gene editing despite the large aliphatic linkage that resulted from the bioconjugation reaction. ${ }^{50}$ Tolerance of Cas 9 to this linkage in the loop adjoining the crRNA and tracrRNA sequences (often referred to as the tetraloop of the sgRNA) is consistent with previous observations that various modifications at the 5 '-terminus of the tracrRNA or tetraloop of the sgRNA did not negatively impact gRNA-Cas9 function. ${ }^{54,55}$ The robust activity of sgRNA modified in this hairpin is also congruent with the crystal structure of Cas9 complexed with a gRNA, showing that the hairpin (tetraloop) connecting the crRNA and tracrRNA sequences protrudes away from the protein. ${ }^{45,56}$ More recently, the Brown group used different azide-alkyne cycloaddition strategies to generate modified sgRNA from two smaller 
fragments. ${ }^{51}$ They explored the impact of different triazole linkages in the tetraloop as well as near the cleavage site across from the 3'-end of the protospacer adjacent motif (PAM) sequence. In both of the bioconjugation reports using $\mathrm{CuAAC}$ to generate the sgRNA, the fragment encompassing the tracrRNA sequence was substantial in length (65 nt, $66 \mathrm{nt}$ or $79 \mathrm{nt}$ ), which is not optimal for solid-phase synthesis. ${ }^{50,51}$

Here we demonstrate that sgRNAs can be assembled from three smaller pieces of RNA, which better leverages the advantages of solid-phase synthesis, and results in a more modular synthetic strategy. This approach uses a middle fragment modified with different fluorophore labels, which is subsequently clicked to a unique crRNA sequence such that each fluorophore corresponds to a specific gene target. We demonstrate that these clicked fluorescent sgRNAs are fully functional in vitro and in cells, although the position of the installed triazole linkage within the tracrRNA does impact efficiency. Moreover, highlighting the utility of this approach, we have shown that two genes can be disrupted in cells with high efficiency when this strategy is used in combination with FACS. We expect this synthetic strategy for the modular assembly of sgRNAs will readily enable access to a variety of chemically-modified or functionalized RNA fragments, facilitating the production of sgRNA libraries for screening and other applications using commercially available solid-phase reagents and simple on-bead protocols.

\section{RESULTS AND DISCUSSION}

Deconstructing the single guide into three similar length fragments, unlike the two fragments strategies previously explored, ${ }^{50,51}$ requires that the Cas9 tolerate unnatural oligonucleotide linkages within the portion of the gRNA corresponding to the tracrRNA sequence that binds Cas9. Therefore, we decided to first explore the impact of introducing an unnatural linkage into the tracrRNA sequence on Cas9 activity, with the motivation of being able to stitch 
together smaller pieces to form the longer, 67 nucleotides, tracrRNA. For our unnatural linkage, we selected the triazole that resulted from the Brown lab's strategy using a copper-catalyzed azidealkyne cycloaddition of a 3'-O-propargyl terminus reacting with a 5'-azide terminated strand (Figure 1). This linkage is remarkably well tolerated by nucleic acid-binding enzymes and can even be read through by some polymerases. ${ }^{57,58}$ Another modification that is well tolerated by Cas9 is the replacement of ribonucleotides with deoxyribonucleotides at several locations in the gRNA sequence. ${ }^{59,60}$ These previous reports suggested that we could use commercially available 3'-O-propargyl-methyldeoxycytosine-modified solid supports to introduce the propargyl functionality and 5'-iodo-modified deoxythymidine to introduce the azide.

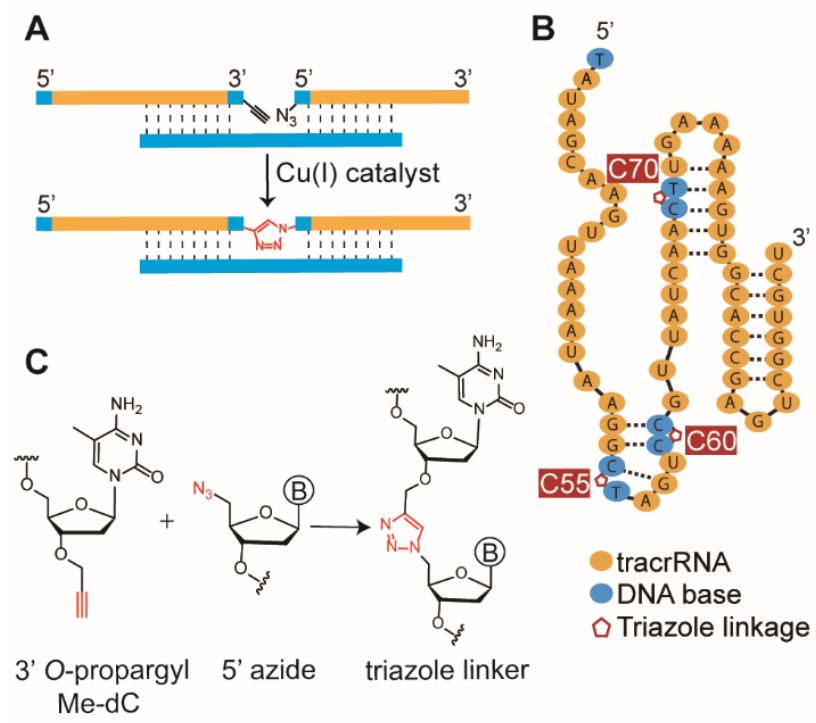

Figure 1. Template-assisted CuAAC assembly of the tracrRNA. (A) Schematic illustrating the DNA-templated copper-catalyzed azide-alkyne cycloaddition (CuAAC) of RNA strands terminated with 3'-O-propargyl and 5'-azido modified deoxyribonucleotides. (B) tracrRNA sequence with the triazole linkages (C70:T71) and DNA substitutions shown. tracrRNA was terminated with a 5'-thymidine to enhance the stability against RNAse degradation. (C) Molecular structure depicting triazole formation from the 5'-azide and 3'- $O$-propargyl Me-dC terminated strands.

We prepared three different lengths of two pairs of RNA strands, 5' azide terminated and 3' propargyl terminated strands that would result in a triazole linkage in tracrRNA strands (Figure 
2A). These RNA strands were synthesized to test the unnatural triazole linkages in various positions in the tracrRNA, including those involved in Cas9 binding (Figure 2B). Two of the 5'azide strands were synthesized using the commercially available 5 '-iodothymidine phosphoramidite followed by nucleophilic substitution with $\mathrm{NaN}_{3}$ prior to base deprotection and cleavage of the DNA-RNA chimera from the solid support. ${ }^{61}$ We also synthesized a strand containing a 5'-azidodeoxyribocytodine (C61) by directly iodinating the 5'-hydroxy group while still on bead. ${ }^{61}$ Introducing the triazoles at these positions allowed us to explore the impact of the triazole linkage location within the tracrRNA on Cas9 activity (Figure 2A, 2B). Our previous work showed that the CuAAC reaction of 5'-azido and 3'-O-propargyl terminated DNA strands proceeds rapidly using a DNA template to bring together the reactive termini in the presence of a $\mathrm{Cu}(\mathrm{I})$ catalyst and 1.5 equivalents (with respect to copper) of a benzimidazole ligand. ${ }^{62}$ Using similar conditions with argon degassing, we observed quantitative conversion of the limiting 3'alkyne oligonucleotide fragment after 1 hour of reaction (Figure S2). After the reaction, the triazole-linked tracrRNAs were purified by polyacrylamide gel electrophoresis and characterized by mass spectrometry. 


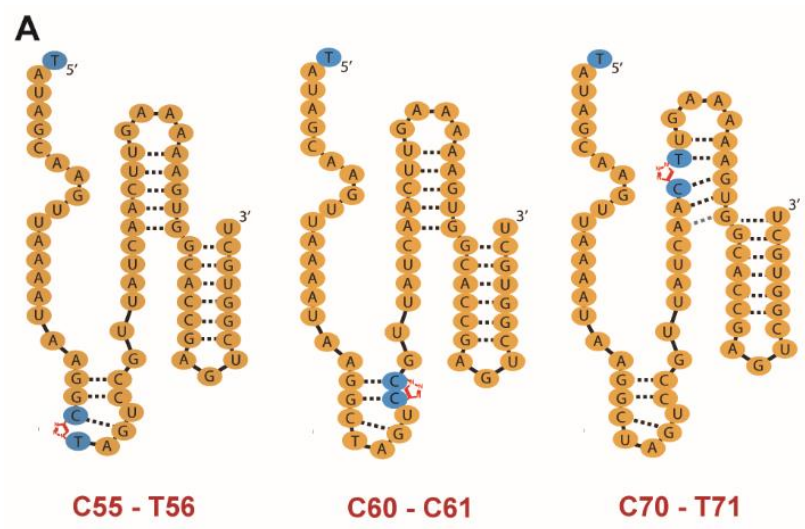

B
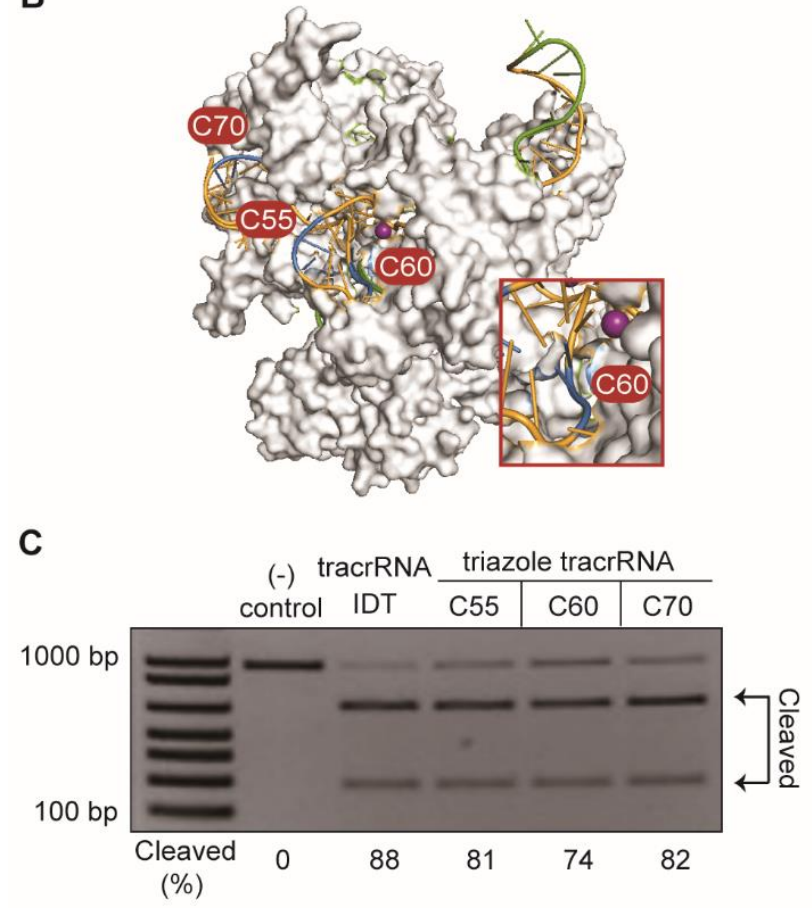

Figure 2. Exploring three different locations for the triazole linkage in tracrRNA. (A) Sequences of the three clicked tracrRNA explored with triazole linkages resulting from 3'-O-propargyl modifications at the C55, C60, and C70, respectively. The tracrRNA was synthesized from two fragments that resulted in a triazole between the C55 and U56 positions. Ribonucleotides are shown in orange and deoxyribonucleotides are shown in blue. The 5'-dT was introduced to increase the stability of the tracrRNA. The crRNA was obtained separately from a commercial source (Integrated DNA Technologies). (B) Structure of Cas9 in complex with a gRNA (PDB 4008) highlighting the position of the three locations where the tracrRNA was clicked together in our studies. (C) Gel electrophoresis image of the in-vitro Cas9 cutting assay with commercially available tracrRNA and triazole-containing tracrRNA at different positions. 
We evaluated the activity of the three triazole-linked tracrRNAs using an in vitro Cas9 cutting assay that can detect DNA target strand cleavage. In this assay, the various triazole-linked tracrRNA were hybridized to a commercial crRNA (from Integrated DNA Technologies; IDT) that targets EMX1 (Figure 2C), complexed with Cas9, then added to a reaction containing amplified DNA corresponding to the EMXI gene target site. We observed cutting of the DNA target for all of the triazole-linked tracrRNAs ( $C 55,81 \%$ and $C 70,82 \%$ cleavage) that was comparable to the results observed for the commercial tracrRNA ( $88 \%$ cleavage). Remarkably, even the tracrRNA with the triazole at the C60:C61 linkage which is in a binding region with the Cas9 nuclease, ${ }^{45}$ exhibited excellent nuclease activity, albeit slightly reduced ( $C 60,74 \%$ cleavage). The appreciable activity for the tracrRNA with the triazole linkage in close proximity to the bound protein provides further support that this specific linkage generated from 3'-O-propargyl and 5'azide nucleotides is accepted by many different enzymes owing to its size and hydrogen-bonding ability that mimic the phosphate linkage. ${ }^{63}$

Having determined that the triazole linkage in the tracrRNA is not deleterious to Cas9 activity, particularly at locations C55:T56 and C70:T71, we next explored the synthesis of sgRNA from three strands. This strategy required the synthesis of a middle sequence that contained both a 5'-azide and a 3'-O-propargyl methyldeoxycytosine. We also introduced an additional dT nucleotide, positioned in the tetraloop region where it can be extended to other applications such as linkage to a fluorophore. All three strands were then clicked simultaneously using two DNA templates to selectively react the desired azide and alkyne termini and prevent unwanted byproducts (Figure 3A). The resulting sgRNA product contained a triazole at the C70:T71 position as well as at the C30:T31 position near the gene targeting sequence (Figure 3B). Progress of the reaction was monitored by denaturing polyacrylamide gel electrophoresis (PAGE), which revealed 
the generation of a larger strand of the expected size of the sgRNA (98mer) (Figure 3C). These modified sgRNAs were purified by extraction from PAGE gels and characterized by electrospray ionization mass spectrometry.

To test whether modified sgRNAs containing two unnatural triazole linkages were active with respect to Cas9, an in vitro DNA cleavage assay was once again performed using the EMX1 target sequence. We compared the in vitro activity of our sgRNA modified with the EMX1 targeting sequence with a scrambled target sgRNA containing the triazole modifications at the same two positions. Excellent activity for the EMXI targeting sgRNA was observed with no activity for the scrambled sgRNA (Figure 3D), indicating that the sgRNA constructed from three smaller fragments maintained its compatibility with the Cas9 enzyme. Next, we evaluated whether our double-clicked sgRNA was active in cells by transfecting Cas9-expressing HeLa cells with a ribonucleotide polymer complex composed of the synthetic sgRNA and Lipofectamine. The target DNA was extracted, and the target region was amplified by PCR. The activity of the synthetically modified sgRNA in cells was measured by the presence of insertions or deletions (INDEL) in the target sequence using the T7 endonuclease I assay. ${ }^{64}$ Despite the presence of two unnatural triazole linkages, we observed the double-clicked sgRNA was active in the unsorted cells after transfection based on the cleavage of the target by T7 endonuclease (Figure 3E). 


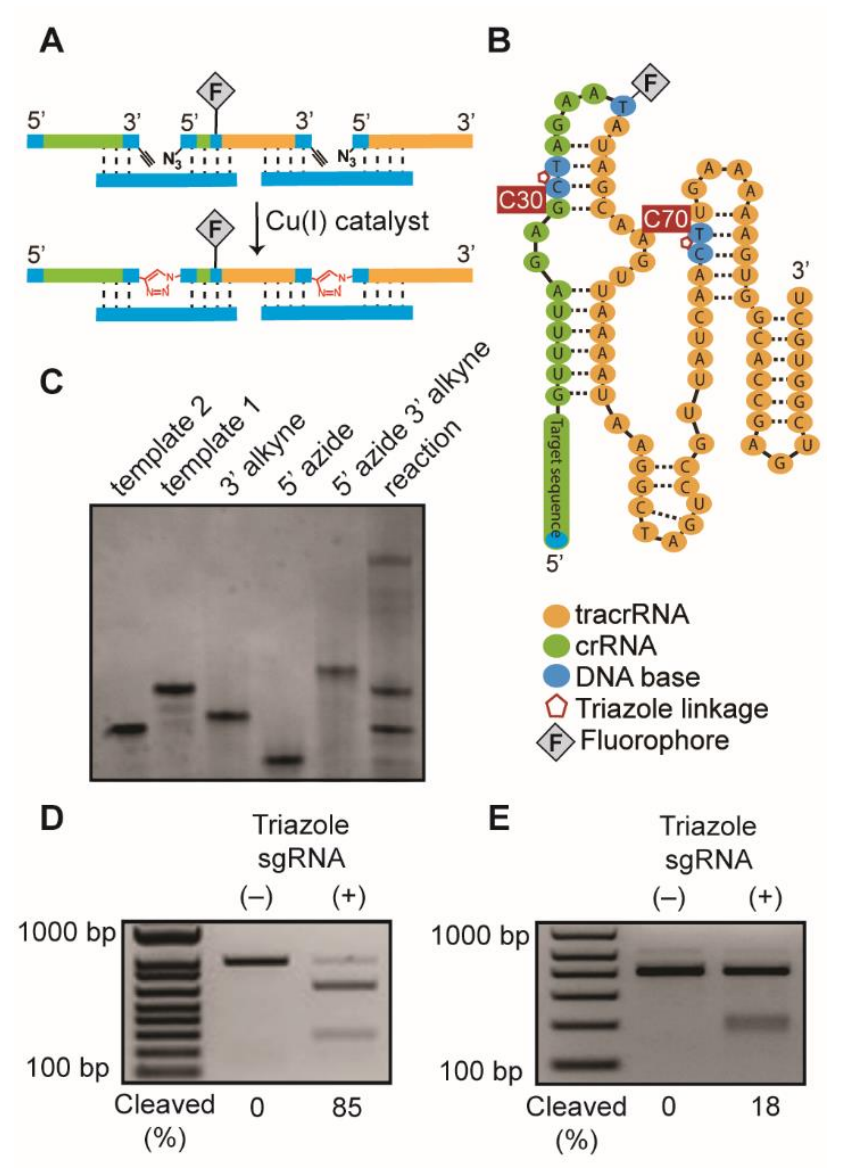

Figure 3. Synthesis and activity of double-clicked sgRNA (A) Reaction scheme illustrating the formation of doubly clicked sgRNA. (B) sgRNA sequence with the functionalized extra dT between A35 and A36. Green represents crRNA, yellow represents tracrRNA, blue represents DNA bases. Triazole linkages are located in between C30:T31 and C70:T71. (C) PAGE image of the double-click reaction. (D) In vitro assay illustrating the activity of the sgRNA targeting EMXI. The control lane (-) is the same experiment using a different sgRNA that did not target the EMXI gene. (E) The T7 endonuclease I assay illustrates cleavage of the EMXI target in unsorted HeLa cells expressing Cas9 after transfection with the EMXI-targeting sgRNA. The control lane (-) is the result of the T7 endonuclease I assay with cells not transfected with the RNP.

One of the major advantages of our approach is that modifications can be incorporated into the fragments while still achieving substantial yields by solid-phase synthesis. Accordingly, we incorporated a fluorescein-modified thymidine to the tetraloop region, adding an additional nucleotide between A35 and A36. To introduce a distinct second label, we prepared a middle fragment with a modified thymidine at the same position that contained a trifluoroacetyl-protected primary amine. After base deprotection and removal from the solid phase, this strand was reacted 
with ATTO 550 NHS ester following standard coupling and purification procedures. The ATTO 550 modified middle fragment was used to prepare the sgRNA targeting EMX1 and the fluorescein modified middle fragment was used for the sgRNA targeting WAS (Figure 4A). These two sgRNAs were transfected into Hela cells stably expressing Cas9, either individually or together using Lipofectamine. Cells were then sorted for the single positive ATTO $550^{+}$or fluorescein ${ }^{+}$ cells for the individual transfections, or the double-positive ATTO $550^{+}$and fluorescein ${ }^{+}$ population from the cells transfected with both sgRNAs (Figure 4B). Most of the cells took up the sgRNAs following transfection as seen by the shift in fluorescence in the appropriate channels (Figure 4C). The top $40 \%$ of the positive cells were sorted in each case. After cell sorting, we quantified the activity of these sgRNAs in cells by measuring cleavage of the target site using the T7 endonuclease I assay (Figure 4D). We found that the ATTO 550-labelled EMX1 sgRNA showed a very similar \% of cleavage in both single sort and dual sort due to high transfection. The fluorescein-labeled WAS sgRNA had higher \% cleavage in single sorting. These results demonstrate that two fluorescently-modified synthetic sgRNAs, produced through a double click reaction, can successfully be transfected into cells to facilitate simultaneous multi-gene editing. 

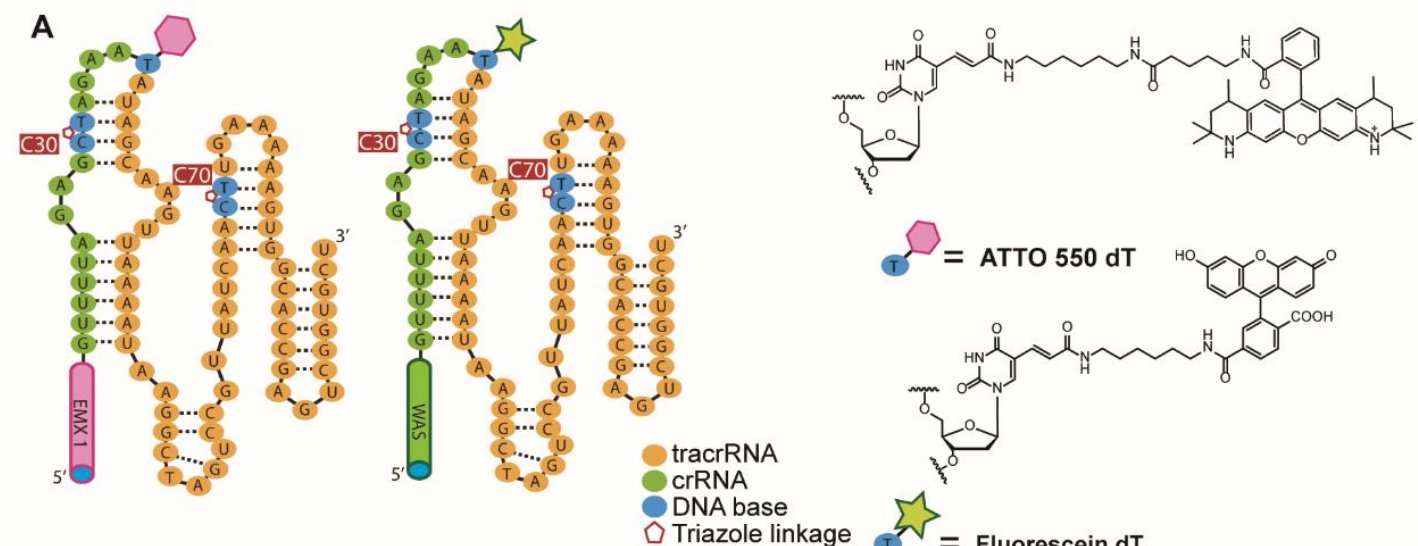

B
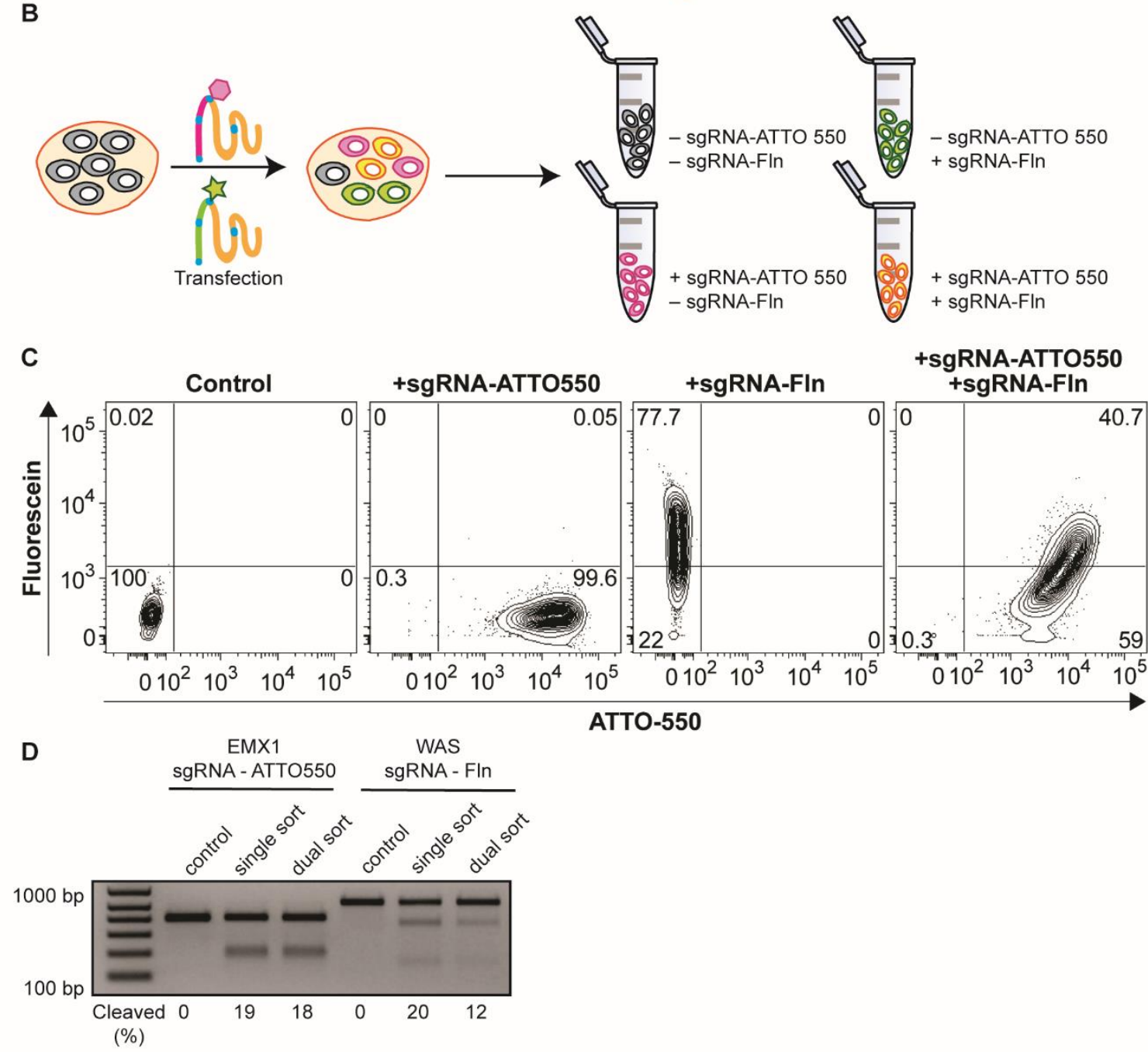

Figure 4. Multiple gene editing using dual fluorescent sgRNA and FACS. (A) sgRNA structures of EMX1 targeting sgRNA labeled with ATTO 550 (pink hexagon) and of WAS targeting sgRNA labeled with fluorescein (green star). (B) Schematic illustrating general cell-based assay with fluorophore-labeled sgRNA. Both sgRNAs were transfected in Cas9 expressing Hela cells followed by FACS sorting based on ATTO 550 and fluorescein emission. Negative control was 
not sorted. (C) FACS results of Hela cells transfected with ATTO 550-labelled EMX1 sgRNA or fluorescein-labelled WAS sgRNA for the single-color transfections and a 50:50 mixture of both sgRNAs for the dual-color transfections. The control cells were not transfected by either sgRNA. (D) Results from the T7 endonuclease I cleavage assay for the EMXI target and WAS target performed on cells. Control - cells not transfected with any sgRNA (unsorted); single sort - cells transfected with either ATTO 550-labeled EMX1 sgRNA or fluorescein-labeled WAS sgRNA and sorted accordingly by FACS; dual sort - cells transfected with both sgRNAs and sorted for both fluorescent colors by FACS.

\section{CONCLUSIONS}

The approach described herein enables the modular and rapid synthesis of fluorescent or other chemically-modified sgRNAs and is scalable to the production of large quantities. By dividing the tracrRNA sequence into two fragments, we were able to generate sgRNA from three fragments of lengths 30, 41, and $27 \mathrm{nt}$. With these lengths, incorporating chemically-modified nucleic acids that improve specificity or guide RNA stability becomes practical. Moreover, our successful implementation of deoxyribonucleic acids at the bioconjugation sites allows anyone with access to a solid-phase synthesizer to make these constructs using commercially available materials and straightforward on-bead reaction protocols. ${ }^{61}$ Thus, this modular bioconjugation strategy of sgRNA can be assembled to achieve multi-color labeling with different fluorophores as well as different chemical modifications. Taken together, this modular strategy of synthesis of sgRNA opens up new possibilities for CRISPR/Cas biotechnological applications such as multigene knockout and imaging.

\section{MATERIALS AND METHODS}

Oligonucleotide (ONs) Synthesis. With the exception of the 63nt DNA ruler that was purchased from Integrated DNA Technologies (IDT), oligonucleotides were synthesized with an Applied Biosystem 394 automated DNA/RNA synthesizer using reagents from Glen Research following 
their recommended procedures. 2'-O-TBDMS phosphoramidites (Bz-A, Ac-G, Ac-C, and U) and 2'-O-Ac-U-CPG were used for the RNA nucleotides. The DNA phosphoramidites utilized were dC, dT, Fluorescein-dT, 5'-iodo-dT, Amino-Modifier C6-dT, 3'-O-5-Methyl-dC Controlled Porous Glass (CPG). To cleave the modified RNA from the solid support, a 1:1 mixture of 30\% ammonium hydroxide (Aldrich 1336-21-6) and $40 \%$ methylamine (Aldrich 74-89-5) was freshly prepared and used following the recommended procedure with an additional AMA incubation of the CPGs for 40 or more minutes. For the 5 ' $-\mathrm{OH}$ terminated strands, we utilized the DMT-on purification protocol with Glen-pak cartridges.

On-CPG 5'-Azide ONs Modification. For the C60 tracrRNA strand, the 5'-hydroxy of the terminal $\mathrm{dC}$ nucleotide had to be iodinated following the literature procedure. ${ }^{61}$ Specifically, after DMT deprotection of the terminal $\mathrm{dC}$ nucleotide, the column containing the CPGs was briefly affixed to the house vacuum to remove the additional solvent. Methyltriphenoxyphosphonium iodide (450 mg) was dissolved in dry DMF ( $2 \mathrm{~mL}, \geq 99.9 \%$ OmniSolv $\left.{ }^{\circledR}\right)$. The solution was transferred into a 1-mL plastic syringe fit. The column containing the CPGs was connected with the syringe containing the iodide solution and an empty $1 \mathrm{~mL}$ syringe and the solution was syringed back and forth for $5 \mathrm{~min}$ and then allowed to stand for 1 hour at room temperature. After an hour, the solution was removed from the CPGs and the CPGs were washed with dry DMF (2-3 mL) and dried on the house vacuum, and immediately used in the next step. The iodo-dT terminated strand made directly from the commercially available phosphoramidite or the iodo- $\mathrm{dC}$ prepared above was used in the next step. A solution of sodium azide (50 mg in $1 \mathrm{~mL}$ dry DMF) was prepared and heated for 10 min at $75^{\circ} \mathrm{C}$ to facilitate dissolution. After brief cooling, the azide solution was taken into a $1 \mathrm{~mL}$ syringe synthesis and introduced to the iodinated strands on the CPGs also utilizing syringesynthetic techniques. The CPGs connected to both syringes were left in the azide solution 
overnight at room temperature. The next morning, the azide solution was removed, the CPGs were washed with dry acetonitrile $(5 \mathrm{~mL})$ and dried on the house vacuum. The 5'-azide ONs was then cleaved from the solid support and further deprotected following the recommended RNA deprotection procedure from Glen Research. To the deprotected 5'-azide ONs, aqueous sodium acetate $(3 \mathrm{M}$, filtered, $25 \mu \mathrm{L})$ and 1-butanol $(1 \mathrm{~mL})$ were added followed by vortexing for 30 seconds. The oligo solution was cooled down to $-20{ }^{\circ} \mathrm{C}$ for $30 \mathrm{~min}$. The precipitated ONs and solution were centrifuged $(12,000 \mathrm{rpm}, 10 \mathrm{~min})$. The supernatant was decanted, and the pellet was washed with ethanol $(95 \%, 0.75 \mathrm{ml})$ twice followed by desalting of the ONs using a Glen-Pak cartridge.

ATTO 550-dT modified ONs. For the 5'-azide, 3'-alkyne modified ONs bearing the internal ATTO 550 label, the Amino-Modifier C6-dT was used in the solid-phase synthesis followed by azide functionalization and strand deprotection and purification as described above. This aminefunctionalized oligonucleotide $(50 \mathrm{nmol})$ was dissolved in a carbonate/bicarbonate buffer $(200 \mu \mathrm{L}$, 0.1 M, pH 9.2). An NHS-ester modified ATTO 550 (Sigma Aldrich cat \# 92835) (350 nmol in 7 $\mu \mathrm{L}$ of DMSO) was added to the oligo solution, mixed well, and left to stand at room temperature for $40 \mathrm{~min}$. After vortexing, the reaction mixture was stored in the refrigerator for 3 hours and then at room temperature for 1 hour. The reaction mixture was desalted with Glen Gel-pak 1.0 desalting column (cat \# 61-5010).

DNA templated CuAAC ligation. DNA-templated CuAAC was performed similar to our previous work. $^{62}$ For triazole tracrRNA synthesis, a solution of 5' azide ONs (30 nmol), 3' alkyne ONs (15 nmol), and DNA Template-1 (15 nmol) was prepared to reach a total volume of $508 \mu \mathrm{L}(0.2 \mathrm{M}$ $\mathrm{NaCl}, 10 \mathrm{mM} \mathrm{MgCl}_{2}$ ). For triazole sgRNA synthesis, 5' azide RNA (20 nmol), 5' azide - 
fluorophore - 3' alkyne ONs (15 nmol), 3' alkyne ONs (20 nmol), DNA Template-1 and -2 (15 $\mathrm{nmol}$ each) were dissolved in a salt solution $(0.2 \mathrm{M} \mathrm{NaCl}, 10 \mathrm{mM} \mathrm{MgCl} 2$ to reach a total volume of $508 \mu \mathrm{L}$. The ONs solution was heated at $80{ }^{\circ} \mathrm{C}$ for $5 \mathrm{~min}$ and cooled down to room temperature for 1 hour to facilitate strand annealing. The ONs solution was then flushed with argon for 2 min.

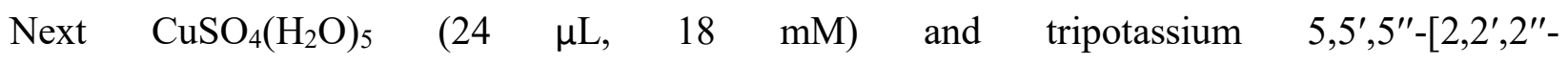
nitrilotris(methylene)tris $\left(1 \mathrm{H}-\right.$ benzimidazole-2,1-diyl)] tripentanoate hydrate $\left(\mathrm{BimC}_{4} \mathrm{~A}\right)_{3}, 36 \mu \mathrm{L}$, $18 \mathrm{mM}$ ) were combined in a small Eppendorf tube, flushed with argon for 2 min and left to sit for $3 \mathrm{~min}$. Next, freshly prepared sodium ascorbate $(24 \mu \mathrm{L}, 340 \mathrm{mM})$ was added to the copper solution followed by flushing with argon for 2 min and left to sit for $3 \mathrm{~min}$. The copper solution (68 $\mu \mathrm{L})$ was added to the ONs solution, followed by argon flush for 2 minutes, then the reaction was incubated at $23{ }^{\circ} \mathrm{C}$ for 2 hours. At this point, the reaction was stopped with the addition of aqueous EDTA (100 $\mu$ L, 0.5 M EDTA) and the solution was lyophilized. The lyophilized reaction was dissolved in $80 \mu \mathrm{L}$ nuclease-free water and $40 \mu \mathrm{L}$ of $0.5 \mathrm{M}$ EDTA. The oligo solution was loaded with $7 \mu \mathrm{L}$ on each lane on a $10 \%$ denaturing PAGE gel $(0.75 \mathrm{~mm}, 10$ wells per gel, 2 gels used for one reaction mixture). The gels were run at $200-250 \mathrm{~V}$ for $60-80 \mathrm{~min}$. Afterward, the gels were visualized by UV or StainsAll (see below). The desired oligo band was cut, crushed, and soaked in nuclease-free water $(800 \mu \mathrm{L})$ and stored in the refrigerator overnight. The oligo solution was filtered by using Spin-X centrifuge tube filter (costar 8160, $0.22 \mu \mathrm{m}$ cellulose acetate) to separate from the crushed gel then desalted by Glen-pack cartridge following the Glen-pak desalting procedure.

Analysis by Polyacrylamide Gel Electrophoresis. A 10\% denaturing polyacrylamide gel $(0.75 \mathrm{~mm}$, 10 wells) was prepared. To analyze the CuAAC reactions, we used StainsAll to visualize each 
ONs band (Aldrich E9379). After electrophoretic separation, the gel was soaked in StainsAll solution (1:1 Formamide/water) (Formamide, Fisher F841) for 10 mins and visualized by white illumination via ImageQuant RT ECL instrument from GE Healthcare life science.

Cloning and plasmid construction. Plasmid templates for in vitro cleavage assays were prepared through ligation of inserts into HindIII/XbaI double-digested pUC19 (ThermoFisher). Singlestranded DNA oligonucleotides containing Cas9 targets were ordered from Integrated DNA Technologies (IDT). Forward and reverse ssDNA oligonucleotides were annealed in a thermocycler by heating to $95^{\circ} \mathrm{C}$ for $5 \mathrm{~min}$, then slowly cooled to $25^{\circ} \mathrm{C}$ over the course of 1 hour prior to ligation.

In vitro cleavage of DNA substrates. In vitro cleavage assays were performed as previously described. ${ }^{35}$ Briefly, primers pUC19_fwd and pUC19_rev were used to generate dsDNA substrates through PCR amplification of previously prepared plasmid templates, followed by purification with the QIAquick PCR Purification Kit (Qiagen). DNA substrate concentration was measured by an Implen NanoPhotomoter NP80 (Implen). Prior to the addition of DNA substrate, Cas9 (NEB) and gRNA were incubated at a ratio of 1:1.5 in Cas9 cleavage buffer $(100 \mathrm{mM} \mathrm{NaCl}, 50 \mathrm{mM}$ Tris$\mathrm{HCl}, \mathrm{pH} 8.0,10 \mathrm{mM} \mathrm{MgCl} 2,100 \mu \mathrm{g} \mathrm{mL}-1 \mathrm{BSA}$ ) for $10 \mathrm{~min}$ at $25^{\circ} \mathrm{C}$ to assemble complexes. For each reaction, $5 \mathrm{nM}$ of DNA substrate was digested with $50 \mathrm{nM}$ Cas9 RNP complex for 1 hour at $37{ }^{\circ} \mathrm{C}$. Reactions were stopped by purifying the cleavage products using the MinElute PCR Purification Kit (Qiagen). The reaction was resolved on a $1.2 \%$ agarose gel, followed by imaging on an Amersham Imager 600 (GE Healthcare). 
Cationic lipid transfection of stable cell lines. Cells stably expressing Cas9 were transfected using Lipofectamine RNAiMAX with sgRNAs according to the manufacturer's instructions to a final concentration of $30 \mathrm{nM}^{8,9}$

Cellular cleavage assays. 48 hours after transfection, genomic DNA (gDNA) was isolated from transfected cells using the DNeasy Blood \& Tissue Kit (Qiagen) and quantified using a NanoPhotometer NP80 (Implen). Locus-specific primers targeting $E M X 1$ and $W A S$, as well as 100 ng of gDNA were used to PCR amplify the target of interest, followed by purification with the QIAquick PCR Purification Kit (Qiagen). T7 endonuclease I (T7E1) digestion was performed using the purified PCR product as described in the manufacturer's protocol (New England Biolabs). Cleavage products were resolved on a 1.2\% TAE agarose gel. For experiments involving the enrichment of fluorescent-positive cells, cells were sorted on a BD FACSAria III instrument 24 hours following transfection by the Flow Cytometry Core at the University of Alberta. PCR amplification and T7E1 digestion was performed as described above. ${ }^{8,9}$

\section{ASSOCIATED CONTENT}

\section{Supporting information}

The supporting information is available free of charge at (link)

Sequence table, mass spectrometry, polyacrylamide gel images.

\section{AUTHOR INFORMATION}

\section{Corresponding Authors}

Julianne M. Gibbs - Department of Chemistry, University of Alberta, Edmonton, Alberta T6G 2G2, Canada. Email: julianne.gibbs@ualberta.ca. 
Matthew S. Macauley - Department of Chemistry and Department of Medical Microbiology and Immunology, University of Alberta, Edmonton, Alberta T6G 2G2, Canada. Email: macauley@ualberta.ca

\section{Authors}

Hansol Park - Department of Chemistry, University of Alberta, Edmonton, Alberta T6G 2G2, Canada

Eiman A. Osman - Department of Chemistry, University of Alberta, Edmonton, Alberta T6G 2G2, Canada

Christopher R. Cromwell - Department of Pharmacology, University of Alberta, Edmonton, Alberta T6G 2G2, Canada

Christopher D. St. Laurent - Department of Chemistry, University of Alberta, Edmonton, Alberta T6G 2G2, Canada

Yuning Liu - Department of Chemistry, University of Alberta, Edmonton, Alberta T6G 2G2, Canada

Elena N. Kitova - Department of Chemistry, University of Alberta, Edmonton, Alberta T6G 2G2, Canada

John S, Klassen - Department of Chemistry, University of Alberta, Edmonton, Alberta T6G 2G2, Canada

Basil P. Hubbard - Department of Pharmacology, University of Alberta, Edmonton, Alberta T6G 2G2, Canada

\section{Authors Contributions}

J.M.G. and M.S.M. conceived the idea and in collaboration with B.P.H. designed the research. H.P., E.A.O., C.R.C., and Y.L. performed the experiments. H.P., E.A.O., C.R.C., C.D.S., and E.N.K. analyzed the data. J.M.G., H.P., M.S.M., and B.P.H. contributed to writing the manuscript. All authors approved the final version of the manuscript.

\section{Notes}

The authors declare no competing interests. 


\section{ACKNOWLEDGMENTS}

M.S.M and J.M.G. acknowledge the New Frontier Research Program for an Exploration grant. H.P. and J.M.G. gratefully acknowledge NSERC CREATE (CREATE-463990-2015) and Deutsche Forschungsgemeinschaft DFG (IRTG 2022) for the Alberta/Technical University of Munich International Graduate School for Hybrid Functional Materials (ATUMS), as well as the University of Alberta Faculty of Science and Faculty of Graduate Studies, Future Energy Systems and the Alberta Innovates Strategic Projects program. M.S.M is supported by a Tier II Canada

Research Chair in Chemical Glycoimmunology. We gratefully thank Gareth Lambkin at the

Biological Services and Jing Zheng at the Mass Spectrometry Laboratory (Department of Chemistry, University of Alberta) for assistance.

\section{REFERENCES}

(1) Jinek, M.; Chylinski, K.; Fonfara, I.; Hauer, M.; Doudna, J. A.; Charpentier, E. A Programmable Dual-RNA-Guided DNA Endonuclease in Adaptive Bacterial Immunity. Science 2012, 337 (6096), 816-821. https://doi.org/10.1126/science.1225829.

(2) Jinek, M.; East, A.; Cheng, A.; Lin, S.; Ma, E.; Doudna, J. RNA-Programmed Genome Editing in Human Cells. eLife 2013, 2, e00471. https://doi.org/10.7554/eLife.00471.

(3) Cong, L.; Ran, F. A.; Cox, D.; Lin, S.; Barretto, R.; Habib, N.; Hsu, P. D.; Wu, X.; Jiang, W.; Marraffini, L. A.; Zhang, F. Multiplex Genome Engineering Using CRISPR/Cas Systems. Science 2013, 339 (6121), 819-823. https://doi.org/10.1126/science.1231143.

(4) Mali, P.; Yang, L.; Esvelt, K. M.; Aach, J.; Guell, M.; DiCarlo, J. E.; Norville, J. E.; Church, G. M. RNA-Guided Human Genome Engineering via Cas9. Science 2013, 339 (6121), 823826. https://doi.org/10.1126/science. 1232033.

(5) Doudna, J. A.; Charpentier, E. The New Frontier of Genome Engineering with CRISPRCas9. Science 2014, 346 (6213), 1258096. https://doi.org/10.1126/science.1258096.

(6) Lin, S.; Staahl, B. T.; Alla, R. K.; Doudna, J. A. Enhanced Homology-Directed Human Genome Engineering by Controlled Timing of CRISPR/Cas9 Delivery. eLife 2014, 3, e04766. https://doi.org/10.7554/eLife.04766.

(7) Tuladhar, R.; Yeu, Y.; Tyler Piazza, J.; Tan, Z.; Rene Clemenceau, J.; Wu, X.; Barrett, Q.; Herbert, J.; Mathews, D. H.; Kim, J.; Hyun Hwang, T.; Lum, L. CRISPR-Cas9-Based Mutagenesis Frequently Provokes on-Target MRNA Misregulation. Nat. Commun. 2019, 10 (1), 4056. https://doi.org/10.1038/s41467-019-12028-5.

(8) Cromwell, C. R.; Jovel, J.; Hubbard, B. P. Methods for Measuring CRISPR/Cas9 DNA Cleavage in Cells. In CRISPR Guide RNA Design; Fulga, T. A., Knapp, D. J. H. F., Ferry, Q. R. V., Eds.; Methods in Molecular Biology; Springer US: New York, NY, 2021; Vol. 2162, pp 197-213. https://doi.org/10.1007/978-1-0716-0687-2_11. 
(9) Cromwell, C. R.; Hubbard, B. P. In Vitro Assays for Comparing the Specificity of Firstand Next-Generation CRISPR/Cas9 Systems. In CRISPR Guide RNA Design; Fulga, T. A., Knapp, D. J. H. F., Ferry, Q. R. V., Eds.; Methods in Molecular Biology; Springer US: New York, NY, 2021; Vol. 2162, pp 215-232. https://doi.org/10.1007/978-1-0716-0687-2_12.

(10) Wang, T.; Wei, J. J.; Sabatini, D. M.; Lander, E. S. Genetic Screens in Human Cells Using the CRISPR-Cas9 System. Science 2014, 343 (6166), 80-84. https://doi.org/10.1126/science.1246981.

(11) Shi, J.; Wang, E.; Milazzo, J. P.; Wang, Z.; Kinney, J. B.; Vakoc, C. R. Discovery of Cancer Drug Targets by CRISPR-Cas9 Screening of Protein Domains. Nat. Biotechnol. 2015, 33 (6), 661-667. https://doi.org/10.1038/nbt.3235.

(12) He, W.; Zhang, L.; Villarreal, O. D.; Fu, R.; Bedford, E.; Dou, J.; Patel, A. Y.; Bedford, M. T.; Shi, X.; Chen, T.; Bartholomew, B.; Xu, H. De Novo Identification of Essential Protein Domains from CRISPR-Cas9 Tiling-SgRNA Knockout Screens. Nat. Commun. 2019, 10 (1), 4541. https://doi.org/10.1038/s41467-019-12489-8.

(13) Schwank, G.; Koo, B.-K.; Sasselli, V.; Dekkers, J. F.; Heo, I.; Demircan, T.; Sasaki, N.; Boymans, S.; Cuppen, E.; van der Ent, C. K.; Nieuwenhuis, E. E. S.; Beekman, J. M.; Clevers, H. Functional Repair of CFTR by CRISPR/Cas9 in Intestinal Stem Cell Organoids of Cystic Fibrosis Patients. Cell Stem Cell 2013, 13 (6), 653-658. https://doi.org/10.1016/j.stem.2013.11.002.

(14) Hsu, P. D.; Lander, E. S.; Zhang, F. Development and Applications of CRISPR-Cas9 for Genome Engineering. Cell 2014, $157 \quad$ (6), 1262-1278. https://doi.org/10.1016/j.cell.2014.05.010.

(15) Dever, D. P.; Bak, R. O.; Reinisch, A.; Camarena, J.; Washington, G.; Nicolas, C. E.; PavelDinu, M.; Saxena, N.; Wilkens, A. B.; Mantri, S.; Uchida, N.; Hendel, A.; Narla, A.; Majeti, R.; Weinberg, K. I.; Porteus, M. H. CRISPR/Cas9 $\beta$-Globin Gene Targeting in Human Haematopoietic Stem Cells. Nature 2016, $539 \quad$ (7629), 384-389. https://doi.org/10.1038/nature20134.

(16) Ye, L.; Wang, J.; Tan, Y.; Beyer, A. I.; Xie, F.; Muench, M. O.; Kan, Y. W. Genome Editing Using CRISPR-Cas9 to Create the HPFH Genotype in HSPCs: An Approach for Treating Sickle Cell Disease and $\beta$-Thalassemia. Proc. Natl. Acad. Sci. 2016, 113 (38), 1066110665. https://doi.org/10.1073/pnas.1612075113.

(17) DeWitt, M. A.; Magis, W.; Bray, N. L.; Wang, T.; Berman, J. R.; Urbinati, F.; Heo, S.-J.; Mitros, T.; Munoz, D. P.; Boffelli, D.; Kohn, D. B.; Walters, M. C.; Carroll, D.; Martin, D. I. K.; Corn, J. E. Selection-Free Genome Editing of the Sickle Mutation in Human Adult Hematopoietic Stem/Progenitor Cells. Sci. Transl. Med. 2016, 8 (360), 360ra134-360ra134. https://doi.org/10.1126/scitranslmed.aaf9336.

(18) Frangoul, H.; Altshuler, D.; Cappellini, M. D.; Chen, Y.-S.; Domm, J.; Eustace, B. K.; Foell, J.; de la Fuente, J.; Grupp, S.; Handgretinger, R.; Ho, T. W.; Kattamis, A.; Kernytsky, A.; Lekstrom-Himes, J.; Li, A. M.; Locatelli, F.; Mapara, M. Y.; de Montalembert, M.; Rondelli, D.; Sharma, A.; Sheth, S.; Soni, S.; Steinberg, M. H.; Wall, D.; Yen, A.; Corbacioglu, S. CRISPR-Cas9 Gene Editing for Sickle Cell Disease and $\beta$-Thalassemia. $N$. Engl. J. Med. 2021, 384 (3), 252-260. https://doi.org/10.1056/NEJMoa2031054.

(19) Leonetti, M. D.; Sekine, S.; Kamiyama, D.; Weissman, J. S.; Huang, B. A Scalable Strategy for High-Throughput GFP Tagging of Endogenous Human Proteins. Proc. Natl. Acad. Sci. 2016, 113 (25), E3501-E3508. https://doi.org/10.1073/pnas.1606731113.

(20) McCarty, N. S.; Graham, A. E.; Studená, L.; Ledesma-Amaro, R. Multiplexed CRISPR 
Technologies for Gene Editing and Transcriptional Regulation. Nat. Commun. 2020, 11 (1), 1281. https://doi.org/10.1038/s41467-020-15053-X.

(21) Wang, H.; Yang, H.; Shivalila, C. S.; Dawlaty, M. M.; Cheng, A. W.; Zhang, F.; Jaenisch, R. One-Step Generation of Mice Carrying Mutations in Multiple Genes by CRISPR/CasMediated Genome Engineering. Cell 2013, 153 (4), 910-918. https://doi.org/10.1016/j.cell.2013.04.025.

(22) Jiang, Y.; Chen, B.; Duan, C.; Sun, B.; Yang, J.; Yang, S. Multigene Editing in the Escherichia Coli Genome via the CRISPR-Cas9 System. Appl. Environ. Microbiol. 2015, 81 (7), 2506-2514. https://doi.org/10.1128/AEM.04023-14.

(23) Sekine, R.; Kawata, T.; Muramoto, T. CRISPR/Cas9 Mediated Targeting of Multiple Genes in Dictyostelium. Sci. Rep. 2018, 8 (1), 8471. https://doi.org/10.1038/s41598-018-26756-z.

(24) Kabadi, A. M.; Ousterout, D. G.; Hilton, I. B.; Gersbach, C. A. Multiplex CRISPR/Cas9Based Genome Engineering from a Single Lentiviral Vector. Nucleic Acids Res. 2014, 42 (19), e147-e147. https://doi.org/10.1093/nar/gku749.

(25) Fu, Y.; Rocha, P. P.; Luo, V. M.; Raviram, R.; Deng, Y.; Mazzoni, E. O.; Skok, J. A. CRISPR-DCas9 and SgRNA Scaffolds Enable Dual-Colour Live Imaging of Satellite Sequences and Repeat-Enriched Individual Loci. Nat. Commun. 2016, 7 (1), 11707. https://doi.org/10.1038/ncomms11707.

(26) Wang, H.; Yang, H.; Shivalila, C. S.; Dawlaty, M. M.; Cheng, A. W.; Zhang, F.; Jaenisch, R. One-Step Generation of Mice Carrying Mutations in Multiple Genes by CRISPR/CasMediated Genome Engineering. Cell 2013, 153 (4), 910-918. https://doi.org/10.1016/j.cell.2013.04.025.

(27) Lim, H.; Choi, S.-K. Programmed GRNA Removal System for CRISPR-Cas9-Mediated Multi-Round Genome Editing in Bacillus Subtilis. Front. Microbiol. 2019, 10, 1140. https://doi.org/10.3389/fmicb.2019.01140.

(28) Zetsche, B.; Heidenreich, M.; Mohanraju, P.; Fedorova, I.; Kneppers, J.; DeGennaro, E. M.; Winblad, N.; Choudhury, S. R.; Abudayyeh, O. O.; Gootenberg, J. S.; Wu, W. Y.; Scott, D. A.; Severinov, K.; van der Oost, J.; Zhang, F. Multiplex Gene Editing by CRISPR-Cpf1 Using a Single CrRNA Array. Nat. Biotechnol. 2017, 35 (1), 31-34. https://doi.org/10.1038/nbt.3737.

(29) Kim, S.; Kim, D.; Cho, S. W.; Kim, J.; Kim, J.-S. Highly Efficient RNA-Guided Genome Editing in Human Cells via Delivery of Purified Cas9 Ribonucleoproteins. Genome Res. 2014, 24 (6), 1012-1019. https://doi.org/10.1101/gr.171322.113.

(30) Filippova, J.; Matveeva, A.; Zhuravlev, E.; Stepanov, G. Guide RNA Modification as a Way to Improve CRISPR/Cas9-Based Genome-Editing Systems. Biochimie 2019, 167, 49-60. https://doi.org/10.1016/j.biochi.2019.09.003.

(31) Mir, A.; Alterman, J. F.; Hassler, M. R.; Debacker, A. J.; Hudgens, E.; Echeverria, D.; Brodsky, M. H.; Khvorova, A.; Watts, J. K.; Sontheimer, E. J. Heavily and Fully Modified RNAs Guide Efficient SpyCas9-Mediated Genome Editing. Nat. Commun. 2018, 9 (1), 2641. https://doi.org/10.1038/s41467-018-05073-z.

(32) Hendel, A.; Bak, R. O.; Clark, J. T.; Kennedy, A. B.; Ryan, D. E.; Roy, S.; Steinfeld, I.; Lunstad, B. D.; Kaiser, R. J.; Wilkens, A. B.; Bacchetta, R.; Tsalenko, A.; Dellinger, D.; Bruhn, L.; Porteus, M. H. Chemically Modified Guide RNAs Enhance CRISPR-Cas Genome Editing in Human Primary Cells. Nat. Biotechnol. 2015, 33 (9), 985-989. https://doi.org/10.1038/nbt.3290.

(33) Basila, M.; Kelley, M. L.; Smith, A. van B. Minimal 2'-O-Methyl Phosphorothioate 
Linkage Modification Pattern of Synthetic Guide RNAs for Increased Stability and Efficient CRISPR-Cas9 Gene Editing Avoiding Cellular Toxicity. PLOS ONE 2017, 12 (11), e0188593. https://doi.org/10.1371/journal.pone.0188593.

(34) Ryan, D. E.; Taussig, D.; Steinfeld, I.; Phadnis, S. M.; Lunstad, B. D.; Singh, M.; Vuong, X.; Okochi, K. D.; McCaffrey, R.; Olesiak, M.; Roy, S.; Yung, C. W.; Curry, B.; Sampson, J. R.; Bruhn, L.; Dellinger, D. J. Improving CRISPR-Cas Specificity with Chemical Modifications in Single-Guide RNAs. Nucleic Acids Res. 2017, 46 (2), 792-803. https://doi.org/10.1093/nar/gkx1199.

(35) Cromwell, C. R.; Sung, K.; Park, J.; Krysler, A. R.; Jovel, J.; Kim, S. K.; Hubbard, B. P. Incorporation of Bridged Nucleic Acids into CRISPR RNAs Improves Cas9 Endonuclease Specificity. Nat. Commun. 2018, 9 (1), 1448. https://doi.org/10.1038/s41467-018-03927-0.

(36) Lee, K.; Mackley, V. A.; Rao, A.; Chong, A. T.; Dewitt, M. A.; Corn, J. E.; Murthy, N. Synthetically Modified Guide RNA and Donor DNA Are a Versatile Platform for CRISPRCas9 Engineering. eLife 2017, 6, e25312. https://doi.org/10.7554/eLife.25312.

(37) Palumbo, C. M.; Gutierrez-Bujari, J. M.; O’Geen, H.; Segal, D. J.; Beal, P. A. Versatile 3' Functionalization of CRISPR Single Guide RNA. ChemBioChem 2020, 21 (11), 16331640. https://doi.org/10.1002/cbic.201900736.

(38) Wang, H.-X.; Song, Z.; Lao, Y.-H.; Xu, X.; Gong, J.; Cheng, D.; Chakraborty, S.; Park, J. S.; Li, M.; Huang, D.; Yin, L.; Cheng, J.; Leong, K. W. Nonviral Gene Editing via CRISPR/Cas9 Delivery by Membrane-Disruptive and Endosomolytic Helical Polypeptide. Proc. Natl. Acad. Sci. 2018, $115 \quad$ (19), 4903-4908. https://doi.org/10.1073/pnas.1712963115.

(39) Wei, T.; Cheng, Q.; Min, Y.-L.; Olson, E. N.; Siegwart, D. J. Systemic Nanoparticle Delivery of CRISPR-Cas9 Ribonucleoproteins for Effective Tissue Specific Genome Editing. Nat. Commun. 2020, 11 (1), 3232. https://doi.org/10.1038/s41467-020-17029-3.

(40) Khan, F. J.; Yuen, G.; Luo, J. Multiplexed CRISPR/Cas9 Gene Knockout with Simple CrRNA:TracrRNA Co-Transfection. Cell Biosci. 2019, $9 \quad$ (1), 41. https://doi.org/10.1186/s13578-019-0304-0.

(41) Rahdar, M.; McMahon, M. A.; Prakash, T. P.; Swayze, E. E.; Bennett, C. F.; Cleveland, D. W. Synthetic CRISPR RNA-Cas9-Guided Genome Editing in Human Cells. Proc. Natl. Acad. Sci. 2015, 112 (51), E7110-E7117. https://doi.org/10.1073/pnas.1520883112.

(42) Karvelis, T.; Gasiunas, G.; Miksys, A.; Barrangou, R.; Horvath, P.; Siksnys, V. CrRNA and TracrRNA Guide Cas9-Mediated DNA Interference in Streptococcus Thermophilus. RNA Biol. 2013, 10 (5), 841-851. https://doi.org/10.4161/rna.24203.

(43) Seki, A.; Rutz, S. Optimized RNP Transfection for Highly Efficient CRISPR/Cas9Mediated Gene Knockout in Primary T Cells. J. Exp. Med. 2018, 215 (3), 985-997. https://doi.org/10.1084/jem.20171626.

(44) Nasri, M.; Mir, P.; Dannenmann, B.; Amend, D.; Skroblyn, T.; Xu, Y.; Schulze-Osthoff, K.; Klimiankou, M.; Welte, K.; Skokowa, J. Fluorescent Labeling of CRISPR/Cas9 RNP for Gene Knockout in HSPCs and IPSCs Reveals an Essential Role for GADD45b in Stress Response. Blood Adv. 2019, 3 (1), https://doi.org/10.1182/bloodadvances.2017015511.

(45) Nishimasu, H.; Ran, F. A.; Hsu, P. D.; Konermann, S.; Shehata, S. I.; Dohmae, N.; Ishitani, R.; Zhang, F.; Nureki, O. Crystal Structure of Cas9 in Complex with Guide RNA and Target DNA. Cell 2014, 156 (5), 935-949. https://doi.org/10.1016/j.cell.2014.02.001.

(46) Finn, J. D.; Smith, A. R.; Patel, M. C.; Shaw, L.; Youniss, M. R.; van Heteren, J.; Dirstine, 
T.; Ciullo, C.; Lescarbeau, R.; Seitzer, J.; Shah, R. R.; Shah, A.; Ling, D.; Growe, J.; Pink, M.; Rohde, E.; Wood, K. M.; Salomon, W. E.; Harrington, W. F.; Dombrowski, C.; Strapps, W. R.; Chang, Y.; Morrissey, D. V. A Single Administration of CRISPR/Cas9 Lipid Nanoparticles Achieves Robust and Persistent In Vivo Genome Editing. Cell Rep. 2018, 22 (9), 2227-2235. https://doi.org/10.1016/j.celrep.2018.02.014.

(47) Yin, H.; Song, C.-Q.; Suresh, S.; Wu, Q.; Walsh, S.; Rhym, L. H.; Mintzer, E.; Bolukbasi, M. F.; Zhu, L. J.; Kauffman, K.; Mou, H.; Oberholzer, A.; Ding, J.; Kwan, S.-Y.; Bogorad, R. L.; Zatsepin, T.; Koteliansky, V.; Wolfe, S. A.; Xue, W.; Langer, R.; Anderson, D. G. Structure-Guided Chemical Modification of Guide RNA Enables Potent Non-Viral in Vivo Genome Editing. Nat. Biotechnol. 2017, 35 (12), 1179-1187. https://doi.org/10.1038/nbt.4005.

(48) Dellinger, D. J.; Timár, Z.; Myerson, J.; Sierzchala, A. B.; Turner, J.; Ferreira, F.; Kupihár, Z.; Dellinger, G.; Hill, K. W.; Powell, J. A.; Sampson, J. R.; Caruthers, M. H. Streamlined Process for the Chemical Synthesis of RNA Using 2'- $O$-Thionocarbamate-Protected Nucleoside Phosphoramidites in the Solid Phase. J. Am. Chem. Soc. 2011, 133 (30), 1154011556. https://doi.org/10.1021/ja201561z.

(49) El-Sagheer, A. H.; Brown, T. New Strategy for the Synthesis of Chemically Modified RNA Constructs Exemplified by Hairpin and Hammerhead Ribozymes. Proc. Natl. Acad. Sci. 2010, 107 (35), 15329-15334. https://doi.org/10.1073/pnas.1006447107.

(50) He, K.; Chou, E. T.; Begay, S.; Anderson, E. M.; van Brabant Smith, A. Conjugation and Evaluation of Triazole-Linked Single Guide RNA for CRISPR-Cas9 Gene Editing. ChemBioChem 2016, 17 (19), 1809-1812. https://doi.org/10.1002/cbic.201600320.

(51) Taemaitree, L.; Shivalingam, A.; El-Sagheer, A. H.; Brown, T. An Artificial Triazole Backbone Linkage Provides a Split-and-Click Strategy to Bioactive Chemically Modified CRISPR SgRNA. Nat. Commun. 2019, 10 (1), 1610. https://doi.org/10.1038/s41467-01909600-4.

(52) Lee, K.; Conboy, M.; Park, H. M.; Jiang, F.; Kim, H. J.; Dewitt, M. A.; Mackley, V. A.; Chang, K.; Rao, A.; Skinner, C.; Shobha, T.; Mehdipour, M.; Liu, H.; Huang, W.; Lan, F.; Bray, N. L.; Li, S.; Corn, J. E.; Kataoka, K.; Doudna, J. A.; Conboy, I.; Murthy, N. Nanoparticle Delivery of Cas9 Ribonucleoprotein and Donor DNA in Vivo Induces Homology-Directed DNA Repair. Nat. Biomed. Eng. 2017, 1 (11), 889-901. https://doi.org/10.1038/s41551-017-0137-2.

(53) Deng, W.; Shi, X.; Tjian, R.; Lionnet, T.; Singer, R. H. CASFISH: CRISPR/Cas9-Mediated in Situ Labeling of Genomic Loci in Fixed Cells. Proc. Natl. Acad. Sci. 2015, 112 (38), 11870-11875. https://doi.org/10.1073/pnas.1515692112.

(54) Briner, A. E.; Donohoue, P. D.; Gomaa, A. A.; Selle, K.; Slorach, E. M.; Nye, C. H.; Haurwitz, R. E.; Beisel, C. L.; May, A. P.; Barrangou, R. Guide RNA Functional Modules Direct Cas9 Activity and Orthogonality. Mol. Cell 2014, 56 (2), 333-339. https://doi.org/10.1016/j.molcel.2014.09.019.

(55) Shechner, D. M.; Hacisuleyman, E.; Younger, S. T.; Rinn, J. L. Multiplexable, LocusSpecific Targeting of Long RNAs with CRISPR-Display. Nat. Methods 2015, 12 (7), 664670. https://doi.org/10.1038/nmeth.3433.

(56) Nishimasu, H.; Cong, L.; Yan, W. X.; Ran, F. A.; Zetsche, B.; Li, Y.; Kurabayashi, A.; Ishitani, R.; Zhang, F.; Nureki, O. Crystal Structure of Staphylococcus Aureus Cas9. Cell 2015, 162 (5), 1113-1126. https://doi.org/10.1016/j.cell.2015.08.007.

(57) El-Sagheer, A. H.; Sanzone, A. P.; Gao, R.; Tavassoli, A.; Brown, T. Biocompatible 
Artificial DNA Linker That Is Read through by DNA Polymerases and Is Functional in Escherichia Coli. Proc. Natl. Acad. Sci. 2011, 108 (28), 11338-11343. https://doi.org/10.1073/pnas.1101519108.

(58) El-Sagheer, A. H.; Brown, T. Efficient RNA Synthesis by in Vitro Transcription of a Triazole-Modified DNA Template. Chem. Commun. 2011, 47 (44), 12057. https://doi.org/10.1039/c1cc14316f.

(59) Rueda, F. O.; Bista, M.; Newton, M. D.; Goeppert, A. U.; Cuomo, M. E.; Gordon, E.; Kröner, F.; Read, J. A.; Wrigley, J. D.; Rueda, D.; Taylor, B. J. M. Mapping the Sugar Dependency for Rational Generation of a DNA-RNA Hybrid-Guided Cas9 Endonuclease. Nat. Commun. 2017, 8 (1), 1610. https://doi.org/10.1038/s41467-017-01732-9.

(60) Yin, H.; Song, C.-Q.; Suresh, S.; Kwan, S.-Y.; Wu, Q.; Walsh, S.; Ding, J.; Bogorad, R. L.; Zhu, L. J.; Wolfe, S. A.; Koteliansky, V.; Xue, W.; Langer, R.; Anderson, D. G. Partial DNA-Guided Cas9 Enables Genome Editing with Reduced off-Target Activity. Nat. Chem. Biol. 2018, 14 (3), 311-316. https://doi.org/10.1038/nchembio.2559.

(61) Sanzone, A. P.; El-Sagheer, A. H.; Brown, T.; Tavassoli, A. Assessing the Biocompatibility of Click-Linked DNA in Escherichia Coli. Nucleic Acids Res. 2012, 40 (20), 10567-10575. https://doi.org/10.1093/nar/gks756.

(62) Osman, E. A.; Gadzikwa, T.; Gibbs, J. M. Quick Click: The DNA-Templated Ligation of 3'- $O$-Propargyl- and 5'-Azide-Modified Strands Is as Rapid as and More Selective than Ligase. ChemBioChem 2018, 19 (19), 2081-2087. https://doi.org/10.1002/cbic.201800305.

(63) El-Sagheer, A. H.; Brown, T. Click Nucleic Acid Ligation: Applications in Biology and Nanotechnology. Acc. Chem. Res. 2012, 45 (8), 1258-1267. https://doi.org/10.1021/ar200321n.

(64) Guschin, D. Y.; Waite, A. J.; Katibah, G. E.; Miller, J. C.; Holmes, M. C.; Rebar, E. J. A Rapid and General Assay for Monitoring Endogenous Gene Modification. In Engineered Zinc Finger Proteins; Mackay, J. P., Segal, D. J., Eds.; Methods in Molecular Biology; Humana Press: Totowa, NJ, 2010; Vol. 649, pp 247-256. https://doi.org/10.1007/978-160761-753-2_15. 